\title{
Preparation of students for tertiary studies requiring mathematics
}

\author{
S. R. Belward* $\quad$ D. F. T. Mullamphy* $\quad$ W. W. Read* \\ G. E. Sneddon*
}

(Received 26 May 2006; revised 13 May 2007)

\begin{abstract}
Mathematics education in Queensland seems to be on a neverending downwards spiral. This is the opinion of the majority of the staff in the School of Maths and Physics at James Cook University. Over recent years the staff in our School have struggled to output students at a standard third year tertiary mathematics level. There are two reasons for this: firstly the intake is from a less well prepared student body and secondly, perhaps more critically, the indicators used to measure the ability of secondary school leavers are most often useless. It is at this lower end where decisions about which subjects a student is to pursue are paramount, both in the short term (success in a semester) and the long term (completion of a degree). In this article
\end{abstract}

*School of Mathematical and Physical Sciences, James Cook University, Townsville, AustraliA. http://www.jcu.edu.au/school/mathphys/staff/maths/Belward.shtml

See http://anziamj.austms.org.au/V47EMAC2005/Belward for this article, (C) Austral. Mathematical Soc. 2007. Published July 17, 2007. ISSN 1446-8735 
we detail what we think is lacking in the preparation of students and demonstrate this by presenting the results of a case study centred on a selection of first year students at James Cook University. We also discuss some of the approaches we have tried in an attempt to overcome these difficulties.

\section{Contents}

1 Introduction

C842

2 Issues we face teaching first year maths

C843

2.1 Weakening entry requirements . . . . . . . . . . . C843

2.2 Lack of consistent background . . . . . . . . . . . . . C844

2.3 Difficulty in use of high school maths grade as predictor of

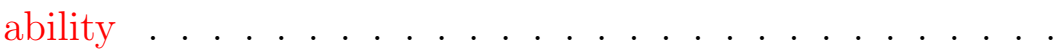

C846

3 Case study

C847

4 Factors leading to the decline

C850

5 Approaches to fixing the problem

C853

5.1 Changes made at the tertiary level . . . . . . . . . C853

5.2 Lobbying to alter methods at the secondary level . . . . . C854

6 Conclusion

C855

References

C856 


\section{Introduction}

Over the last 10 to 15 years there has been a steady decline in the mathematical ability of entering students to the Faculty of Science, Engineering and Information Technology (FSEIT) at James Cook University (JCU). This decline has been observed in differing ways. The most obvious evidence is obtained by comparing the performance of students on final exams, and the format of the examinations themselves, in the main-stream first year mathematics subjects over the time period above. Despite the fact a rigorous introduction to calculus and linear algebra has given way to a "hand-waving" glance at the most important results, students struggle with material that in the past was second nature on entry to the University.

It is important to note that this feeling of inadequate numeracy skills on arrival to the FSEIT is one that is not solely discussed within the School of Mathematical and Physical Sciences, but rather it is discussed over the Faculty as a whole. Most disciplines within FSEIT require Queensland Mathematics B (Maths B) as a pre-requisite for entry to their major. Maths B is regarded across the State as the standard preparation for disciplines that require numeracy skills on arrival at university. Unfortunately the feeling amongst staff within FSEIT is that mainstream secondary level mathematics is not preparing students adequately for tertiary level studies offered within FSEIT.

To compound matters we find that the background of students who have completed Maths B is inconsistent, in particular the grade a student receives is not a reliable indicator of knowledge of basic skills. The issue of inhomogeneous student backgrounds and how these are dealt with in the tertiary sector is a topic that has received wide attention in the tertiary education community for at least 20 years. For example, Blyth, Calegari and Kurz [1, 2] detail remedial courses in Australia and Germany aimed at students with varying backgrounds and more recently the proceedings of Delta conferences contain many articles discussing this issue. While we do discuss our approach to 
dealing with the cohort of inhomogeneous students, this article focusses on the methods used to prepare and grade students for tertiary mathematics.

Section 2 discusses the issues faced within FSEIT at JCU. Our belief is that we are not the only tertiary institution suffering in this way. After discussions with lecturers external to JCU we are now certain that many regional and metropolitan universities in Australia face similar problems. Section 3 presents a "case study" indicating how our sense of frustration with this issue arises. The reasons behind the decline in student ability are discussed in Section 4. Section 5 briefly discusses some of the approaches we used to overcome the difficulties we face, while Section 6 makes concluding comments. We also suggest that the School of Mathematical and Physical Sciences at JCU may contribute to a broader debate on the problem of lower levels of mathematical ability found by most universities in Australia.

\section{Issues we face teaching first year maths}

The decline in the basic mathematical ability of new first year students at JCU is caused by several factors. These include weakening entry requirements and the lack of a consistent mathematics background from secondary school. These problems are compounded by the fact that high school grades are not a strong indicator of likely success at standard first year mathematics. Most of these issues are not unique to JCU, however the severity of the problems at JCU may be greater than in some stronger southern universities. We discuss each one of these issues in the following.

\subsection{Weakening entry requirements}

The push for additional money, and hence more students, has resulted in some universities lowering entrance requirements and enrolling students who 
are ill-prepared for tertiary study. This is especially the case when it comes to mathematics. At JCU it is now possible to enrol in a Bachelor of Science without having studied senior mathematics (such as Maths B) at secondary school. The expectation is that these students will pick up a Maths B "look alike" on entry to the University. Despite the best efforts of University staff, it is not possible to cover the material learnt over a period of two years in Maths B, in one introductory mathematics subject of 39 lectures. The desperate "grab" for students has resulted in JCU and similar universities being forced to allow inappropriate short-cuts in the preparation of students for tertiary studies requiring mathematics. Even students who indicate that they wish to complete a mathematics major at JCU may be accepted into the programme without Mathematics B as a prerequisite.

In addition to the relaxation of Maths $\mathrm{B}$ as a prerequisite for entry to the BSc, JCU has followed the lead of all other Queensland universities and no longer has any course requiring the more challenging Mathematics $\mathrm{C}$ (Maths C) as a prerequisite for entry. From the perspective of an upper secondary student there is no obvious need to study Maths C, as it is not a prerequisite for any university course anywhere in Australia. This has had the disastrous impact of taking Maths C off the list of "must do" subjects for those wishing to enrol in engineering or physical sciences at tertiary level.

In general, students arrive at JCU with weaker ability in mathematics than they did even just five years ago. The University clearly condones this, by its own entry requirements. This cannot be helpful at a time when, in so many of the disciplines JCU aspires to lead, there is a great need for numeracy skills.

\subsection{Lack of consistent background}

Despite the fact that Mathematics B is not a prerequisite for study in many courses, there are still large numbers of students entering JCU having com- 
pleted it. This is because there is still a perception that there is an advantage in having some numeracy skills on entry to university. Indeed there is still some weight given to this in the procedure for allocating places in certain university courses. We are also fortunate that Maths B is still a formal prerequisite to the Bachelor of Engineering degree.

Unfortunately it appears that students completing Maths B lack a consistent background. It is difficult to pin-point the exact reason for these differences. The Mathematics B Syllabus [3] details "subject matter" that can be assumed to be covered in the course. The majority of this subject matter is consistent with what we would expect students to have some familiarity with on arrival at university. Our belief is that the expectation that the subject matter be framed in modelling and problem solving scenarios is the largest contributor to inconsistency in Maths B.

The syllabus clearly states four objectives it aims to address. These are, "knowledge and procedures", "modelling and problem solving", "communication and justification" and "affective". The first objective addresses the need to be competent in algebra, recall definitions and results and to select and use appropriate mathematical procedures. Note that the approach used to gain these skills is well understood and documented. For example there are countless textbooks addressing these issues, so teaching these skills should not be a problem, time permitting. Students who satisfy the knowledge and procedures objective have the mathematical skills required for study in the disciplines that prescribe Maths B as a prerequisite for entry to university, which is all most staff in FSEIT require.

The second objective is by far the most difficult to accomplish, but for teachers who wish to showcase the applicability of mathematics this is the one most tempting to address. For example students are expected to demonstrate "forming a mathematical model of a life-related situation". This is a skill difficult to learn, even after completing undergraduate mathematics at university. Unfortunately the list of sub-objectives within the modelling and problem solving objective is almost twice as long as the list in the knowledge 
and procedures objective. This leads teachers to spend more time trying to satisfy each of these tasks, with the assumption that knowledge and procedures can be learnt along the way. Note also that the approach used to gain modelling and problem solving skills is not well defined. In fact the syllabus specifically states "The learning experiences are suggestions only and are not prescriptive. Schools are encouraged to develop further learning experiences ...". We argue that allowing the most difficult aspect of Maths B to be poorly defined is a big mistake. This is especially so in an environment where so many high school mathematics teachers do not have a mathematics major prior to obtaining a teaching qualification. All these factors inevitably lead to variability in the student experience in Maths B.

A typical intake into first year at JCU comprises students from many secondary schools across Queensland. The difficulty teachers have in covering the more challenging objectives of the curriculum has lead to a vast array of experiences under the Maths B label. This variability in background makes the job of building on this material at university a difficult task. Unfortunately this job is made even harder because the measures used to describe a student's ability in Maths B are inaccurate as we describe in the next Section.

\subsection{Difficulty in use of high school maths grade as predictor of ability}

There are five grades a student can obtain in Maths B and a result of "Sound Achievement (SA)" is usually considered to be a pass in the subject. However a student entering the main first year mathematics subject, MA1000, from Maths B, with only a grade of SA will usually struggle to pass. This is probably because SA can correspond to an overall mark for the subject of as low as $40 \%$.

These factors are compounded by the lack of a consistent background across the student cohort as we discussed above. Not surprisingly, a student's 
result in Maths B is not a good indicator of their likely performance at university. This is confirmed by our data which we describe in the next section.

At JCU the situation has been recognised as being so bad that students entering with a SA from Maths B may now elect to repeat the material by enrolling in the JCU "Preparatory Mathematics" subject, the Maths B looka-like subject we described in Section 2.1. The unfortunate aspect is that while many students with SA in Maths B struggle with MA1000, there are some who can cope with the material, so we cannot make a simple statement that all students with SA in Maths B must revise the material.

The frustration of the lack of a reliable measure of suitability to subjects requiring competency in mathematics is experienced by both staff and students. As students enter first year, decisions are made regarding which subjects, majors or degree they are suitable for. Unfortunately some students have inflated expectations as they have arrived with a label of SA in Maths B and may be permitted to enrol in subjects they are not suited to. This is a diabolical situation as students are given false hope on the basis of a poorly measured grade in Maths B. It is at this lower end that reliability in the Maths B grade is desperately needed. A semester of bad experiences at university may well result in a dissatisfied student leaving. In the current climate this is something we desperately have to try to avoid.

\section{Case study}

We now present a brief analysis of the mathematical ability of MA1000 students (Townsville Campus) of study period 1, 2005. In this class of roughly 200 students, 140 had completed Maths B, the remainder coming from interstate, or overseas, or completing alternate preparatory subjects. The analysis shown here is centred on the students who completed Maths B as preparation for MA1000. 
Students completing Maths B are issued one of five grades. From lowest ability to highest, these are Very Low Achievement (VLA), Low Achievement (LA), Sound Achievement (SA), High Achievement (HA) and Very High Achievement (VHA). Students with grades of VLA or LA are not permitted to enrol in MA1000.

The analysis has two aims. The first is to measure ability at basic algebra and analysis - skills normally accessible to students mid-way through secondary school. The second aim is to gain some idea as to the likely success or otherwise of Maths B graduates at MA1000. In each case the analysis is split according to leaving grade from Maths B. Thus in each case there are three categories, one for each of SA, HA and VHA.

In the first week of lectures the students were presented with a diagnostic test, un-announced, covering simple algebra from middle secondary school. An example of the sort of questions that were asked is:

Expand brackets and collect like terms to simplify the expression

$$
(x-3)^{2}-\left(x^{2}-9\right) .
$$

The test comprised of short answer questions and was marked out of 20 . Figure 1 shows a box plot of the results of the test. The "box" on each grade represents the middle $50 \%$ of the data, while the solid line in each box represents the median of the scores in that group. The lines extending from each box represent the upper and lower quartiles, with individual outliers being marked separately in each category.

Figure 1 clearly shows that more than $25 \%$ of students obtaining SA in Maths B obtained less than half marks on the diagnostic test, while nearly $75 \%$ of those receiving $\mathrm{SA}$ and $50 \%$ of those receiving HA gave incorrect responses to more than one quarter of the questions. Given that the test was comprised of relatively simple middle secondary algebra and analysis, 


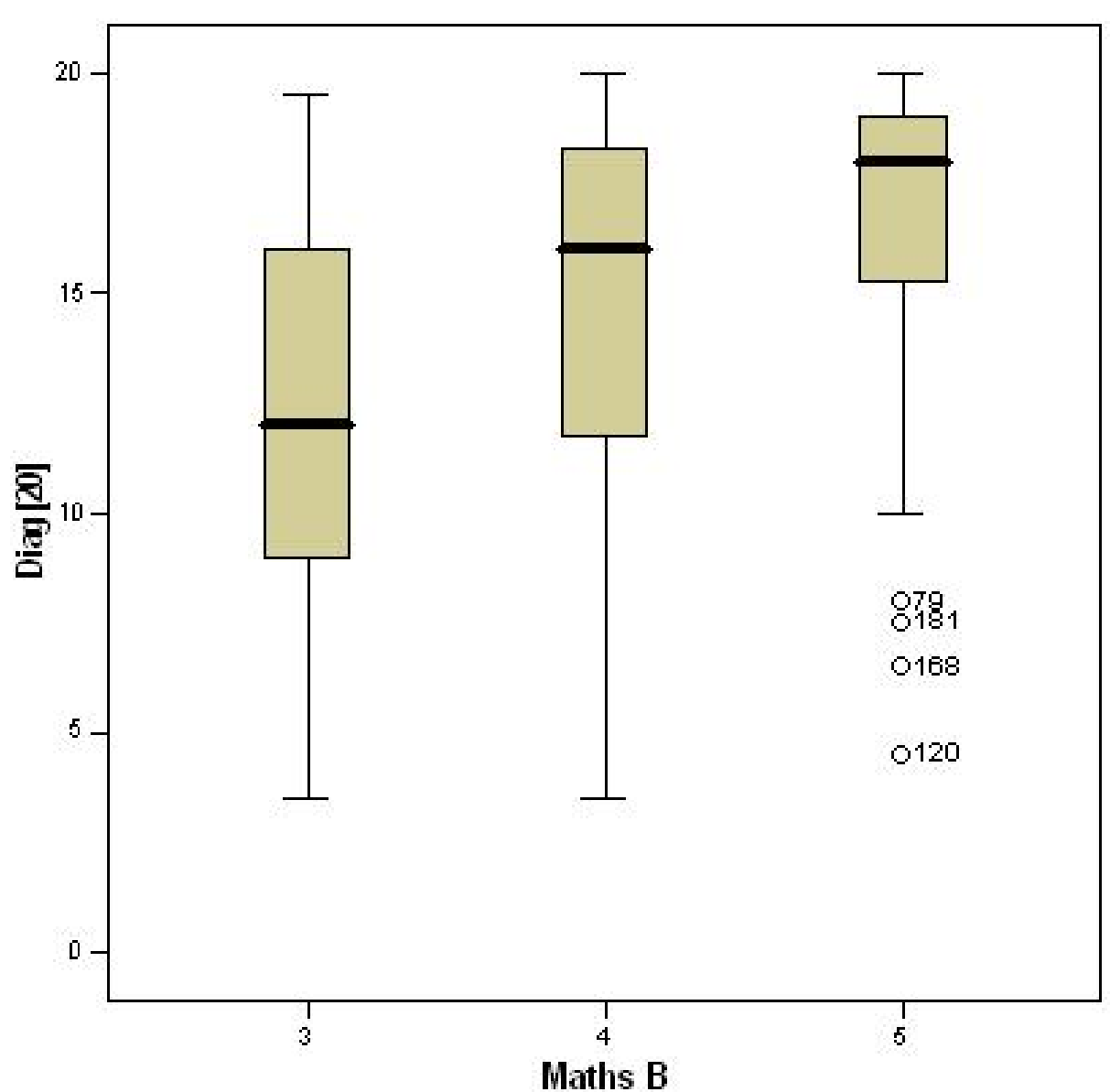

FiguRE 1: A box-plot showing performance on diagnostic test on entry to MA1000. The vertical axis shows scores out of 20 while the horizontal axis shows grades in Maths B: "3" corresponds to "Sound Achievement", "4" corresponds to "High Achievement" and "5" corresponds to "Very High Achievement". 
these results begin to highlight the difficulty we have relying on material we previously would have expected as second nature on entry to university.

Figure 2 shows box plots of the final score in MA1000. This figure serves to highlight the difficulty entering students encounter when they attempt MA1000. The material is not difficult, $80 \%$ comprising of elementary algebra and calculus much of which is contained within the Maths B syllabus. For example a typical question is:

Find the partial fraction expansion for $\frac{5 x+1}{x^{2}-x-12}$. Use this to calculate $\int \frac{5 x+1}{x^{2}-x-12} d x$

More than $75 \%$ of SA students obtain less than $50 \%$ in the subject while barely half of all HA students obtain $50 \%$. The only group for which success is far more likely than failure is the VHA group where more than $75 \%$ of students obtain more than $50 \%$ of the marks.

Together Figures 1 and 2 show that students with SA in Maths B are destined to struggle with MA1000; however, there are a significant proportion who can obtain a passing grade. This again serves to highlight the difficulty we having placing students into the appropriate maths course on arrival at JCU.

\section{Factors leading to the decline}

In this section we briefly discuss the reasons behind the failure of Maths B to deliver students who have the mathematical skills required for success in engineering and science and in particular the failure of these students to cope with continuing maths subjects at university. Some of these issues have been addressed in Section 2. In particular we have described how the 


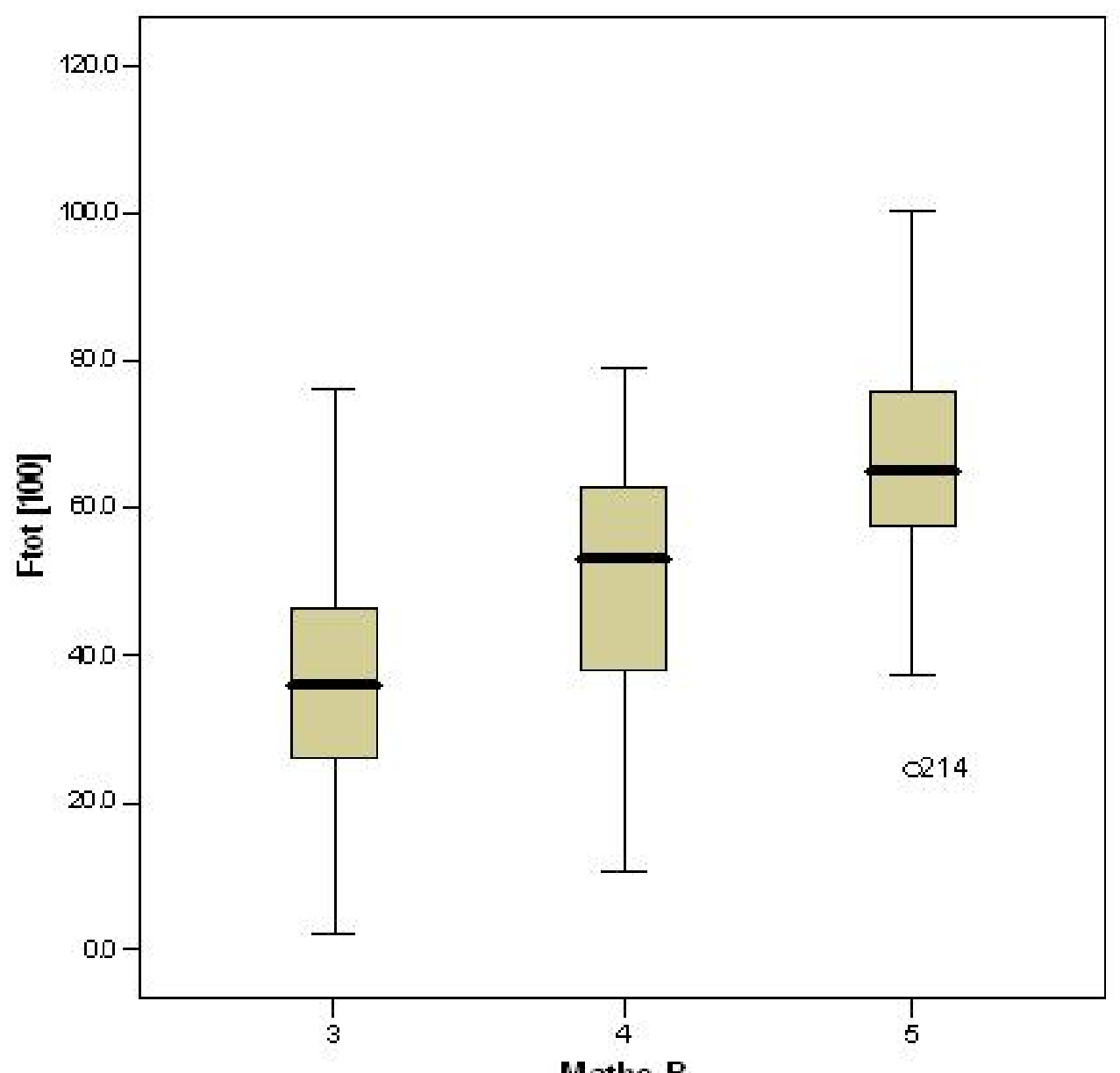

Maths B

Figure 2: A box-plot showing performance in MA1000. The vertical axis shows scores out of 100 while the horizontal axis shows grades in Maths B: "3" corresponds to "Sound Achievement", "4" corresponds to "High Achievement" and "5" corresponds to "Very High Achievement". 
University system itself contributes to the decline in standards by lowering entry requirements.

We have also discussed the over-ambitious nature of the Math B curriculum and how that removes time for students to familiarise themselves with basic definitions and algebraic procedures. The consensus within the School of Maths at Physics at JCU is that there is insufficient emphasis on practice in high school mathematics. Although we are in favour of showing how mathematics can be used in modelling and problem solving, this should not come at the expense of practice.

The diagnostic test discussed in Section 3 also confirmed our belief that errors such as

$$
(x-y)^{2}=x^{2}-y^{2} \quad \text { and } \quad \frac{1}{a+b}=\frac{1}{a}+\frac{1}{b}
$$

are commonplace. The common thread across a large proportion of the student body is that every function is linear, that is,

$$
f(a+b)=f(a)+f(b), \quad \text { irrespective of the function, } f .
$$

Simple rearrangement of formulae also causes problems for many students and has been a source of frequent complaints from outside mathematics, in science and engineering disciplines where Maths B is a pre-requisite.

Unfortunately the situation appears to be worsening. The reliance on calculators is escalating so that instead of just "number sense" diminishing, skills in algebra are on the decline. This is partly because symbolic manipulation is now performed on many calculators. The typical argument given is that this now allows students an opportunity to explore the applications of mathematics, rather than be concerned with algebraic detail. Unfortunately this statement is flawed, one cannot imagine a pianist not practicing their scales. Basic algebra skills are necessary to give insight and a feel for the sorts of possibilities any given mathematical formulation may allow. This will aid any problem solving or modelling scenario. Any perceived gain in 
using symbolic manipulation packages is quickly lost if the user does not have a feel for the underlying mathematics.

As commented in Section 2.1 the Maths B syllabus contains the material we need covered in order for students to progress within FSEIT. Unfortunately these rather simple objectives have been overtaken by the glory of modelling and problem solving. The educational theory that context-based learning is useful because it motivates students is almost certainly true. Unfortunately in Maths B we have seen the ambition of the educational theorists overtake the needs of the end user. A measured use of context-based learning is needed and this cannot come at the expense of fundamentals.

\section{Approaches to fixing the problem}

Over recent years we have been proactive in addressing the problems associated with our weaker student intake into mathematics. We are not interested in "sitting in our ivory tower" and complaining - we have a genuine interest in fixing the problem. Our approach to dealing with the problem has been two-fold. Initially we addressed the issue by altering the content and method of delivery, while more recently we have attempted to form partnerships with local school teachers to influence the content and delivery of material at the secondary level.

\subsection{Changes made at the tertiary level}

We have been willing to adapt to the abilities of our student base and numerous attempts have been made to cater for the weaker mathematical abilities of incoming students. In the mid nineties the pass rates were abysmal and, rather than fail most of the students, a decision was made to create a weaker course to accommodate them. The result was the MA1000 subject we see 
today. This led to the "dumbing down" of the other mainstream first year mathematics subject, and part of the second year program. There have been strong objections to any further drop in standards, so alternative strategies have been employed. These include changing the format of tutorials, running revision tutorials (with free coffee and biscuits) and allowing re-sits on sets of key "proficiency tests". These tests highlighted the algebraic skills we have tried to foster. However, as the proficiency tests were of a very simple nature, their use as a reliable assessment tool was questionable and their effect on student attitudes was also detrimental. These conclusions were similar to those made by Blyth and Calegari [1] who conducted simple objective tests and stated that the questions "were so trivial that students gained entirely the wrong attitudes with respect to the standard and nature of the work required and examined". In 2005 we introduced on-line quizzes in MA1000. Again these are designed to examine basic skills, and can be used as regularly as a student wishes. Unfortunately, while some of these initiatives have been enjoyed by students, the general trend of weaker students on entry to MA1000 has not been countered.

\subsection{Lobbying to alter methods at the secondary level}

Rather than just reacting to the output from the secondary system by altering what we do at the tertiary level, we have been proactive in trying to influence the teaching of secondary mathematics through direct communication with mathematics teachers in secondary schools. This has taken several forms.

One of the authors regularly attends the meetings of the Townsville Branch of the Queensland Association of Mathematics Teachers (QAMT). This gives an opportunity for the university to provide feedback directly to the school teachers. This partnership has also led to JCU mathematicians giving talks at the local schools as well as attending activities such as maths camp. By showing an interest in this way we hope to build a relationship that can be used to express our point of view. 
The Townsville Branch of the QAMT also hosts an annual conference for mathematics teachers. JCU mathematics staff regularly speak at this conference and this exercise has proven to be very effective in expressing our opinion. However, this must be done carefully so as not to set up an "us and them" scenario where the teachers feel they are getting "told off" by a group of arrogant academics. We have had some success at these meetings and although discussions have been robust, there still exists a significant number of teachers sympathetic to our ideas.

In the last two years, 2005 and 2006, the School of Maths and Physics has formed a partnership with some Catholic secondary schools allowing us to deliver MA1000 to high achieving students studying both Maths B and Maths C. This scenario is seen to be mutually beneficial as it enables us to have direct input into the mathematics being taught at these schools whilst allowing the schools to use the formal partnership with JCU as a marketing tool. This partnership has received significant media coverage and has proven to be very successful with interest expressed from schools as far away as Mackay and Cairns. This "jockeying for position" amongst competing high schools has proven to be beneficial to us as it keeps the discussion on standards strongly in the public eye.

The nature of this lobbying means that significant time is required before any substantial benefits can be observed. We cannot expect an immediate turn around in methods and content at high schools, so patience is required on our behalf. This is very much a "work in progress"; however, the benefits of more uniform and robust algebraic skills should be worth the wait.

\section{Conclusion}

In this article we have discussed the "state of play" in first year mathematics at JCU. There is general dissatisfaction with numeracy skills of entering students, including those who have completed the recommended pre-requisite 
for science and engineering, Maths B. A case study was conducted to measure the ability of students who have completed Maths B, and then to examine the success of these students as a function of leaving grade from Maths B. This study showed that more than $75 \%$ of students who obtained the lowest passing grade in Maths B failed to obtain $50 \%$ of the marks in MA1000.

It is our belief that the factors leading to the decline in student ability include a lack of reinforcement of basic skills. This is in part due to the desire to place the subject in context, which is often difficult to do convincingly in mathematics. We have attempted to address this issue by altering the delivery of our subject material. A longer term, but potentially more beneficial approach has been the formation of partnerships between mathematicians and high school teachers.

As mentioned in Section 2 we are certain that JCU is not the only university where declining numeracy skills are having an impact. These problems may not be so prevalent in the more prestigious "sandstone" universities; however, we expect that most regional universities and a significant proportion of metropolitan universities are in a similar situation to JCU. At JCU the feeling is that there is little else we can do on our own to address the problems we face. We are looking to forming partnerships with other deliverers of first year mathematics subjects who feel the same way. Our hope is that a united voice across Australia will have some influence in to what is happening in secondary school mathematics.

\section{References}

[1] W. F. Blyth, and J. P. Calegari, "A multi-level entry mathematics course with remedial component", Int. J. Math. Educ. Sci. Technol. 17:465-472, 1986. doi:10.1080/0020739860170408 C842, C854 
[2] G. Kurz, "Remedial courses in mathematics - Scopes and problems - a survey for the Federal Republic of Germany", Int. J. Math. Educ. Sci. Technol. 16:211-225, 1985. doi:10.1080/0020739850160212 C842

[3] Mathematics B Senior Syllabus 2001, Queensland Board of Senior Secondary School Studies. http:

//www.qsa.qld.edu.au/yrs11_12/subjects/maths_b/syllabus.pdf C845 